\title{
Paideia y Gestión: \\ Un Ensayo sobre el Soporte Paideia en el Trabajo en Salud
}

\author{
Paideia y Gestión: \\ An Essay about Holding in Health Work
}

Gastão Wagner Sousa Campos ${ }^{1}$

1Profesor titular del
Departamento de Medicina
Preventiva. Universidad
Estadual de Campinas.
UNICAMP. Brasil.
gastaowagner@mpc.com.br
RESUMEN Este ensayo presenta una función gerencial original denominada Soporte Paideia. Se trata de una metodología constructiva para ampliar la capacidad de análisis y de intervención de equipos y gerentes de salud en situaciones complejas, y en la elaboración de planes, proyectos de intervención sobre procesos salud-enfermedad individuales o colectivos, y evaluación de acciones de salud. Esta metodología considera los efectos interactivos de los recursos de poder y de conocimiento, como así también los efectos de los afectos sobre el trabajo y la gestión en salud.

PALABRAS CLAVE Gestión en Salud; Funciones Gerenciales; Salud Pública.

\begin{abstract}
This essay presents a management function named Paideia Holding. It is about a methodology able to increase the capacity of health teams and managers to deal with complex situations. A constructive conception is suggested to build plans, therapeutics projects and evaluation of health care. With that purpose, resources of power, knowledge and emotion are analyzed and used.
\end{abstract}

KEY WORDS Health Management; Management Functions; Public Health. 


\section{ACERCA DEL CONCEPTO SOPORTE}

El Soporte Paideia es una postura metodológica que busca reformular los tradicionales mecanismos de gestión. No se trata de una propuesta que busque suprimir otras funciones gerenciales, sino un modo complementario para realizar la coordinación, planificación, supervisión y evaluación del trabajo en equipo. En general, estas funciones son ejercidas con un importante grado de distancia entre los ejecutores de las funciones de gestión y los operadores de actividades finales.

En resumen, se podría afirmar que el recurso de Soporte procura escapar a la tendencia común de varias escuelas de gerencia que intervienen sobre los trabajadores y no de manera interactiva con ellos. En realidad, se considera que el saber gerencial no escapó a la tendencia predominante en la ciencia de suponer una relación casi aséptica entre aquellos que ejercen funciones de conducción y los ejecutores de tareas. Una reiteración de la separación entre el sujeto del conocimiento y del poder y el objeto a ser conocido y manipulado $(1,2)$.

El Soporte parte de la presuposición que las funciones de gestión se ejercen entre sujetos, aunque con distintos grados de saber y de poder.

Por otro lado, asume que todo trabajo tiene una triple finalidad y produce efectos en tres sentidos distintos: primero, objetiva e interfiere con la producción de bienes o servicios para personas externas a la organización -se trabaja para un otro en referencia a los equipos de operadores-; segundo, procura siempre asegurar la reproducción ampliada de la propia organización; y tercero, termina interfiriendo en la producción social y subjetiva de los propios trabajadores y de los usuarios. La gestión tradicional, al lidiar con los trabajadores y con los usuarios como objeto, centra los esfuerzos en las dos primeras finalidades, descuidando la tercera (3).

En realidad, al no reconocer que toda gestión es producto de una interacción entre personas, con frecuencia se verifica una tendencia a la reproducción de formas burocratizadas de trabajo, con empobrecimiento subjetivo y social de los trabajadores y de los usuarios. El Soporte Paideia procura compatibilizar estas tres finalidades, reconociendo que la gestión produce efectos sobre los modos de ser $y$ de proceder de los trabajadores y de usuarios de las organizaciones.

En este sentido, el Soporte Paideia reúne una serie de recursos metodológicos que permiten lidiar con estas relaciones entre sujetos de un modo diferente. Un modo interactivo, un modo que reconoce la diferencia de roles, de poder y de conocimiento, pero que busca establecer relaciones constructivas entre los distintos actores sociales. Así, la supervisión y evaluación deberían comprometer a los propios evaluados tanto en la construcción de los diagnósticos como en la elaboración de nuevas formas de accionar; o sea, formas democráticas para coordinar y planificar el trabajo. Formas que aprovechen y consideren la experiencia, el deseo y el interés de sujetos que no ejercen funciones típicas de gestión. El Soporte Paideia depende de la instalación de alguna forma de cogestión.

El término Soporte pretende capturar todo este significado: no se trataría de comandar objetos sin experiencia o sin interés, sino de articular los objetivos institucionales a los saberes e intereses de los trabajadores y usuarios. Tampoco se apuesta solamente a los recursos internos de cada equipo. El término Soporte indica una presión desde afuera, implica traer algo externo al grupo que opera los procesos de trabajo o que reciben bienes o servicios. Quien es soporte sustenta y empuja al otro. Siendo, en consecuencia, sustentado y empujado a su vez por el equipo "objeto" de la intervención. Todo junto y al mismo tiempo.

Sería importante elaborar también algunos comentarios acerca del sentido de la metodología Paideia.

\section{EFECTO PAIDEIA ¿Cómo provocar el efecto Paideia en personas y agrupaciones?}

Antes que nada, ¿qué es el Efecto Paideia? El término Paideia es de origen griego, y dentro del período clásico designaba uno de los componentes considerados esenciales de la democracia ateniense. Existía el concepto de ciudadanía, que aseguraba derechos a aquellos considerados personas; el de Ágora, que indicaba un espacio y un modo para el ejercicio del poder 
compartido entre gobernantes y ciudadanos; y existía el concepto de Paideia, que significaba la necesidad de educación integral de las personas, creándose un proceso continuo de ampliación de la capacidad de participar de la vida social, inclusive ocupando un lugar en el Ágora (4). En este caso, se realiza una adaptación del concepto original, conservándose, no obstante, sin el sentido principal. En esta metodología de trabajo con grupos y equipos, se considera el Efecto Paideia como un proceso social y subjetivo en que las personas amplían su capacidad de buscar informaciones, de interpretarlas, buscando comprenderse a sí mismas, a los otros y al contexto, aumentando, en consecuencia, la posibilidad de accionar sobre estas relaciones.

Existen tres formas básicas de accionar: las prácticas sociales -algunas estructuradas, como la clínica, la pedagogía, el trabajo en general, y otras características de la vida cotidiana, como las relaciones amorosas, familiares, etc.-, la política y la gestión de colectivos organizados. Nótese que existe una graduación de escalas entre estas formas de accionar sobre el mundo: las políticas se refieren a la dimensión macrosocial, las prácticas a lo micro, y la gestión sería un modo de mediar entre estas esferas. Ciertamente existe comunicación e interferencia entre estas esferas: una condicionando, y produciendo las otras. En este sentido, habría que trabajar con la concepción de inter-producción; o sea, la gestión o las prácticas nunca serán sólo un desdoblamiento de la política, existe una autonomía relativa de cada una de esas esferas. Dicho de otra manera, existe una dependencia relativa entre cada una de esas dimensiones.

El Método Paideia se aplica a la política de posición, buscando evitar la guerra de movimiento. Antonio Gramsci adaptó estos conceptos, oriundos de la estrategia militar, para repensar modos de hacer política donde la construcción de consensos negociados resulte en mayor beneficio y menor daño a la sociedad. Según él, en la guerra de movimiento se anhelan cambios mediante la destrucción de los adversarios, y en política existen momentos en que situaciones de esta índole se imponen a los actores sociales. Sería el caso de la guerra civil, donde se avanza hacia la eliminación completa del poder del adversario, algunas veces con la muerte de millares de personas. Existen, también, formas más civilizadas de políticas de enfrentamiento, por ejemplo, en elecciones democráticas: aunque no esté prevista la eliminación física del opositor, los electores están obligados a elegir entre personas y proyectos distintos. El juego es uno u otro, estando, por lo tanto, dentro de la lógica de la lucha de movimiento. En la política de posición se busca la composición de intereses y de proyectos, y el resultado es considerado positivo cuando se logra el establecimiento de contratos sociales que incluyan intereses de segmentos y clases menos favorecidas por los contratos anteriores (5). El Método Paideia es útil para la construcción de políticas de posición.

En regímenes democráticos, una vez pasadas las elecciones, los plebiscitos y otras situaciones de confrontación, se torna necesaria la gestión de las instituciones según la nueva correlación de fuerzas. En estos casos, manteniéndose la estrategia de guerra de movimiento, se avanza hacia situaciones que paralizan la vida social. En realidad, se podría Ilegar a la conclusión de que si en la política existen inevitables situaciones donde diferentes bloques se enfrentan, obligando a la sociedad a escoger entre una u otra alternativa, en la gestión y en el trabajo es siempre más adecuado buscar estrategias de posición, creando mecanismos para que los conflictos se expresen, pero, al mismo tiempo, para que exista construcción de contratos y de compromisos entre los distintos actores. En este sentido, el Método Paideia es particularmente útil para pensar la gestión de las más diversas prácticas sociales. Para que el statu quo no se mantenga, para aumentar el poder de contratación de los menos favorecidos, es necesario que estos segmentos amplíen su capacidad de comprenderse a sí mismos insertos en el mundo y que se valgan de esa comprensión para tomar decisiones y para intervenir sobre las relaciones sociales.

Comprenderse implica analizar la dinámica del deseo y de los intereses personales; visualizando, al mismo tiempo, el deseo y el interés de los otros, todo esto inmerso en una dinámica histórica y social: la materialidad de las necesidades y el poder de las instituciones. Ahora, esto es tan sólo una descripción sofisticada del simple ejercicio de la vida humana; o sea, vivir es realizar estas operaciones de forma más o 
menos consciente. Estamos siempre inmersos en esta dinámica, estamos siempre aprendiendo de la vida.

El Método Paideia es un reconocimiento de este hecho, pero es también una tentativa de sistematizar modalidades de intervención de forma deliberada en esa dinámica. Pensar y accionar con deliberación es actuar según finalidades, buscando algún sentido para la vida. El Efecto Paideia ocurre sin que tomemos conciencia, aprendemos viviendo. Sin embargo, se puede buscar este efecto según valores e intenciones previas. Reconociendo que el control sobre este proceso será siempre parcial, ya que existen, al mismo tiempo, innumerables factores produciendo efectos sobre personas e instituciones.

La humanidad ya "inventó" varios métodos para interferir en ese proceso: la política, la gestión, la regulación y el control, la pedagogía y la clínica son algunos ejemplos.

El control social sobre las personas es el resultado de la continua producción interactiva de afectos, de conocimientos y de poder. La política enfatiza modalidades de operar con el poder; la pedagogía, la propaganda, los medios de comunicación y la informática, con el conocimiento; las terapias de la subjetividad, las religiones y el arte, con el afecto. Estos campos disciplinarios separan estas tres dimensiones cuando en realidad actúan de forma conjunta, simultánea. La constitución de sujetos, de las necesidades sociales y de las instituciones es producto de las relaciones de poder, del uso de conocimientos y de modos de circulación de afectos.

El Método Paideia es el reconocimiento de que somos producidos de esa forma. Sin embargo, es también el reconocimiento de que cada sujeto, cada colectivo, puede valerse de esas líneas de fuerza para construir nuevas instituciones, nuevas necesidades sociales y nuevas personas. Para esto necesitamos de una pedagogía, de medios de comunicación, de instituciones comprometidas con el Efecto Paideia y no solamente con el control social. Esto sólo será posible mediante la democratización de esas instituciones, o sea, mediante su reforma por una política de posición.

El Método Paideia es una tentativa de pensar reformas y de accionar considerando estas tres dimensiones. Y más aún, acrecienta una finalidad adicional a toda agrupación humana: la del desarrollo de sus integrantes según estas tres perspectivas; o sea, es el esfuerzo por construir una nueva capacidad de pensar y de accionar, sea colectivamente o de cada una de las personas involucradas. La novedad es la integración de estas tres dimensiones: la libertad, o la ciudadanía, o la salud, todo depende de la capacidad de lidiar con los afectos, con saberes y con el poder.

En toda institución ocurren múltiples síntesis de los efectos de esos tres factores; sin embargo, la racionalidad gerencial hegemónica piensa la gestión de organizaciones como una continuidad del poder político, y como un espacio donde los patrones de conocimiento y de circulación de afectos considerados adecuados estarían fijos. Los gestores intentan articularlos para aumentar el control sobre los sujetos. En realidad, se construyó el imaginario de que la gestión no interferiría con estas dimensiones, apenas administraría lo dado a priori: la gerencia sería un desdoblamiento "natural" del poder constituido, un poder delegado por la propiedad privada o por el Estado; el conocimiento sería traído hacia las organizaciones desde afuera, inyectado por medio de cursos de formación regular u otras formas de capacitación y de asesoría; en cuanto a los afectos, cabría a los sujetos "adaptarse" a la lógica de cada organización, acomodándose a las relaciones de poder y protegiéndose de la competencia, de los conflictos, etc.

El Método Paideia imagina contaminar la política, la gestión, la pedagogía, la clínica y la salud pública con la lógica de esta triple determinación: en todas estas formas de accionar sobre el mundo se mezclan poder, saber y afectos. Vale señalar que esa mezcla sucede de formas diferentes, algunas veces una dimensión predominando sobre la otra. En general, se puede afirmar que el accionar político tiene como núcleo específico el tema del poder, es en su campo de fuerzas donde circulan saberes y afectos. La pedagogía tiene como núcleo lo cognitivo, la propaganda, mientras que los medios tienen como núcleo la comunicación y el convencimiento, y en ese campo es que giran el poder y los afectos. Las terapias de la subjetividad y el arte tienen en el núcleo los afectos y en el campo, el poder y lo cognitivo. 
Es interesante resaltar que en varias prácticas sociales, particularmente en aquellas constituidas a partir de una pretensión exagerada de objetividad, ni siquiera se admite que habría influencia de factores políticos, cognitivos o afectivos. La Gestión, la Salud Pública y la Clínica, por ejemplo, operan como si hubiese una dilución de la influencia de estos factores; el saber de los técnicos actuaría mejor "libre" de la política, del conocimiento no científico o de afectos "inconvenientes". Este es el núcleo del pensamiento tecnocrático: la tentativa de reducir personas a la condición de objeto, rechazando la circulación de afectos y la "contaminación" de las prácticas por el poder o por el saber de los "laicos", todos alcanzados por las medidas generadas en apenas uno de los polos de la relación de poder (6).

\section{RECURSOS METODOLÓGICOS PARA EL SOPORTE PAIDEIA ¿Cómo operar con la fun- ción de Soporte Paideia?}

En primer lugar, involucra el intento de ampliar la capacidad de las personas para lidiar con el poder, con la circulación de afectos y con el saber. Lidiar mientras están haciendo cosas, trabajando, cumpliendo tareas. En el fondo, es una forma de radicalizar la construcción de ciudadanía y sociedades democráticas.

El lugar de Soporte no es un lugar organizacional específico, aunque pueda serlo eventualmente (en la Secretaria de Salud de Campinas/SP, en el año 2000, optamos por cambiar el nombre y el modo de actuar de los antiguos supervisores de distrito y de programas, denominándolos soportes institucionales). En el ejercicio de cualquier rol dentro de un colectivo se puede actuar según la perspectiva de Soporte Paideia. Esto es posible, aunque deba considerarse la función de cada uno en la estructura de poder, como así también el dominio de teorías y de métodos y las relaciones de afecto, ya que todo eso influencia fuertemente la capacidad y las posibilidades de soporte; o sea, existen variaciones obligatorias en el modo de proceder según el lugar institucional del supuesto soporte. Consideremos algunos lugares institucionales desde donde se puede realizar Soporte Paideia: $\rightarrow$ desde un lugar de poder institucional: un dirigente de una organización, de un equipo o de un movimiento social puede, sin abandonar su rol, ser soporte de sus dirigidos. ¿Cómo un director, un supervisor o un auditor conseguirían ampliar su modo de actuación, no sólo controlando, fiscalizando y dando órdenes, sino dando soporte a sus supervisados?

$\rightarrow$ desde un lugar de supuesto saber: alguien externo a la agrupación, alguien que es procurado en función de un presunto conocimiento (asesor, consultor, alguien con dominio de un método, o de alguna experiencia previa, analista o soporte institucional) podría accionar, esencialmente, valiéndose de la metodología de soporte.

$\rightarrow$ desde un lugar de supuesto saber $y$, al mismo tiempo, de poder institucional: un profesional (clínico, pedagogo, sanitarista, etc.) puede ejercer sus funciones específicas modificándolas en función de ofrecer soporte a los usuarios.

$\rightarrow$ desde un lugar de paridad (supuesta horizontalidad) desde el colectivo: un miembro de un equipo o de la comunidad, al participar de espacios de cogestión e incluso en el cotidiano, puede funcionar como soporte Paideia para sus pares.

Ciertamente, existen diferencias en el modo de realizar el soporte si se ocupa un rol de director, de soporte externo, de operador de punta o de miembro de un equipo. Los dirigentes tienen más poder, pero en compensación están condicionados por un mayor compromiso con la eficiencia y eficacia organizacional. Siempre que ingresan a una rueda lo hacen con ese sesgo. Esto es un problema, pero, al mismo tiempo, enriquecen al grupo al aportar a la rueda temas del contexto y otros específicos de su función. Un jefe mantiene su poder aun cuando se incorpore a una rueda de discusión; esto no anula la eficiencia de ese mecanismo de gestión.

Soportes externos (consultores, analistas, etc.) tampoco son neutros: en general, traen un referencial teórico y una experiencia que condicionan su visión. Esto no debe ser silenciado; al contrario, debe ser examinado y analizado, ya que puede ser un elemento enriquecedor para el grupo y para los dirigentes. Por otro lado, en 
general, están menos condicionados que los dirigentes y miembros del equipo en las disputas internas por el poder y por la circulación viciada de afectos. Pueden ayudar al grupo a visualizar y a trabajar situaciones problemáticas fundadas en esa dinámica.

Los operadores de punta, en general, y particularmente en el área de servicios, salud y educación, tienen contacto directo con los usuarios. ¿Cómo ser soporte de comunidades, pacientes o alumnos? Ciertamente, no renunciando al saber técnico o a la necesidad de intervenir, pero, tal vez, interactuando con los usuarios tanto en el momento del diagnóstico del problema, como en la definición del proyecto de intervención. Lo fundamental, en ese caso, es mantener siempre la preocupación de aumentar la autonomía de los usuarios, o sea, ampliar la capacidad de autocuidado por parte de ellos, tanto elevando su capacidad de comprender el problema en cuestión como de accionar sobre él.

Por último, cómo ejercer la función de soporte en una situación de paridad: el soporte al propio equipo de trabajo, o, siendo usuario, el soporte a otros miembros de la comunidad y de los propios equipos. Aquí está la esencia de la democratización de las instituciones, ya que eso sólo ocurrirá cuando exista la posibilidad de que todos ejerzan la gestión. En el fondo, aunque la función principal de esos sujetos no sea la gerencia, se parte del supuesto de que participar de la gestión forma parte del campo de responsabilidades de todos los implicados en un proceso de trabajo. Al integrarse a un grupo, un consejo, o una asamblea, es fundamental la postura de abrirse a la influencia de los otros sin renunciar a los intereses y las experiencias personales. Desarrollar capacidad para construir análisis y soluciones compartidas. Combinar firmeza con apertura a la creación de intereses y de una visión del mundo. Capacidad para reconocer autoridad en otros sin someterse a ella. Reconocer el límite impuesto por el colectivo sin desistir de la singularidad de sus deseos y de concepciones.

Enumeremos algunos recursos metodológicos útiles para la función de soporte.

PRIMERO: construir ruedas. Siempre, en todas estas posibilidades, el Soporte debe contribuir activamente a la creación de espacios colectivos; o sea, de escenarios o dispositivos (settings) que propicien la interacción inter-sujetos. Más aún, espacios donde el análisis de situaciones y la toma de decisiones sean posibles. Instituir el hábito de evaluar los procesos y las tareas, redefiniéndolos conforme lo acordado. Resumiendo: el objetivo de esas ruedas es la construcción de Proyectos de Intervención; o sea, se analiza para decidir y definir contratos que se desdoblan en responsabilidades y tareas. La evaluación del resultado de las intervenciones es fundamental para "abrir" posibilidades y superar los conflictos institucionales. Una especie de planificación constructiva y progresiva.

SEGUNDO: incluir en el análisis las relaciones de poder, de afecto y la circulación de conocimientos. El Soporte y el proceso de Soporte también deben ser sometidos a evaluación y corrección de rumbo. Así, el diagrama de relaciones de cada organización debe ser llevado a la rueda. El Soporte debe permitir, acordar y hasta buscar activamente incluirse en esos procesos; o sea, sufrir el Efecto Paideia. Para esto es fundamental desmentir la falacia de la neutralidad, explicitando el lugar y el modo de vinculación de cada uno. Lo que significa analizar las relaciones de poder, de conocimiento y de afecto involucradas. Los analistas institucionales denominan este proceso de análisis de implicancia. Y esto no tiene por qué ocurrir de un modo salvaje, todo al mismo tiempo, transparencia total desde el comienzo. No, jesto sería un desastre! El grado de profundización de los temas se subordina a la tarea y al propio grupo, lo que es o no necesario y soportable en cada momento. Esto se descubre en aproximaciones sucesivas.

TERCERO: traer hacia el trabajo de coordinación, planificación, supervisión y evaluación la lógica de la triple finalidad de las organizaciones. Analizar los procesos según los tres objetivos básicos, a saber: calificar el accionar institucional para el cumplimiento de finalidades externas al grupo -producir valores de uso para otros-, para la defensa y reconstrucción de la propia organización, y para asegurar el desarrollo y la realización personal y profesional del grupo receptor del soporte. Operar con esa lógica amplía la comprensión y la capacidad operacional de los 
colectivos. Los trabajadores, los militantes de un movimiento, todos son medios para conseguir algún resultado, pero, al mismo tiempo, son también un fin de todo y cualquier emprendimiento humano. Esta es la clave de seguridad, sin la cual ni se puede comenzar a realizar la función de Soporte Paideia.

La vida es lo real, concreto y ocurre en el medio, ya que el comienzo o el origen son puro recuerdo -historia-, y el futuro es fantasía pura. No se está aquí negando la importancia de la historia o de las utopías y de los sueños; lo que se afirma es la importancia de valorizar las potencialidades del presente. Sin esta directriz -es en el presente que se analiza y se decide-, no existe el soporte Paideia, aunque pueda ocurrir una serie de efectos interesantes.

Ciertamente, personas con mayor poder institucional, dirigentes, gerentes, tienen dificultades adicionales para ser coherentes con esta directriz. Su papel estructural los condiciona a valorizar la producción de valores de uso en detrimento de los intereses de los trabajadores. Además tienden a reducir el coeficiente de análisis, ya que están preocupados con la conservación de su propio poder. No obstante, se puede recrear la lógica de estos lugares con el objetivo de constituir sujetos autónomos, ya que operadores más analíticos suelen ser, en general, más eficaces, una vez que se comprometen de un modo creativo con la producción de valores de uso.

CUARTO: Trabajar con una metodología dialéctica que traiga Ofertas externas y que al mismo tiempo valorice las Demandas del grupo. El Soporte debe construir y acercar a las ruedas sugerencias en cuanto a modos de analizar y de intervenir sobre la vida. Además de esto, debe recoger las Demandas del grupo y de aquellos con que está interactuando. $\mathrm{Y}$ aquí la primera Oferta es ofrecerse como Soporte, no hay cómo escapar de esto. Explicitar: existe un método, existen temas prioritarios, hay no dichos, hay resistencias, bloqueos, hay correlación de fuerzas, existe la necesidad de establecer contratos con otros intereses, otras culturas, etc. $Y$ toda oferta es una oferta interesada, revela las relaciones de poder, afectivas y los campos de conocimiento del Soporte. Ofertar es revelarse, es exponerse a la evaluación ajena, es un ejemplo
Paidéia, un estímulo para superar la inercia del grupo. Nadie se sale de sí, tirándose de los pelos como "el varón mentiroso".

Por otro lado, las demandas del grupo funcionan como ofertas para el soporte, son los escalones desde donde partir hacia el establecimiento de nuevos compromisos y contratos, comprometerse con los otros y consigo mismo.

Sería importante profundizar métodos para ofertar cosas y para incorporar las demandas del equipo y de los usuarios. Existen tres campos desde donde se originan las ofertas y las demandas: uno relativo a lo que viene desde fuera de las organizaciones, o sea, a la producción de valores de uso y a las formas de comprometerse con los otros. El segundo se refiere a las instituciones, el contexto donde se trabaja. En estos casos, el soporte debe ofertar y sustentar el debate sobre modelos de atención en salud, modelos pedagógicos, formas de organizar el trabajo, los movimientos, etc. Pero comprometerse de forma crítica, tensando límites, explorando posibilidades de realizar tareas de un modo diferente. El tercer campo es el relativo a la ampliación de la autonomía del grupo, aprender a circular, a transitar, crear alas, depender menos del soporte. Y aquí otra directriz Paideia: el soporte funciona solamente cuando el grupo consigue ampliar sus referencias, y más aún, cuando consigue lidiar con estas referencias de un modo crítico, o sea, como otras ofertas; o sea, con generosidad crítica; o sea, sin adhesión automática y sin paranoia.

QUINTO: apoyar al grupo tanto para construir Objetos de inversión como para crear compromisos y contratos con otros. La vida, mientras, ocure: sin disfrutar el cotidiano, no hay sentido para la vida. La postura burocrática trascurre, en gran medida, apostando todo al futuro. La construcción de objetos de inversión exige que el grupo se autorice a ser feliz, a realizarse personal y profesionalmente en el contexto real en que trabajan. El resentimiento o la culpa, la no apropiación de nuevos conocimientos, la concentración de poder, todos son factores o síntomas que impiden la constitución de objetos de inversión, y, en estos casos, el efecto Paideia se cumple de forma precaria, limitada. Una de las tareas del soporte se refiere exactamente a capacitar a las agrupaciones para que depositen afectos positivos en 
objetos (cosas) fuera de sí mismos, aunque referenciadas por el deseo e interés de cada uno. Sentir placer por lo que se hace, apostar a proyectos, en la construcción de nuevas relaciones de afecto y de poder. Disfrutar el placer de saber. La construcción de este objeto de inversión depende de factores políticos, cognitivos y afectivos. Hay que lidiar con esta mezcla.

Por otro lado, accionar en función de otros, estableciendo compromisos y contratos; o sea, sin renunciar de modo alguno al deseo o al interés particular; muy por el contrario, accionar teniéndolos como referencia, para crear espacios y modalidades de acción que establezcan mediaciones entre el interés de los usuarios y el principio de realidad, el contexto histórico y social.

SEXTO: Pensar y hacer junto con las personas, $y$ no en lugar de ellas: para que esto ocurra no alcanza con que el soporte imagine que sabe cuáles serían los contratos o los objetos de inversión más adecuados. Es importante que procure saber sobre esto, sin lo cual no sabrá cómo realizar ofertas. Mientras tanto, lo importante es que dé soporte al grupo para que ellos sepan sobre esto, a la manera de ellos, dialogando con el supuesto saber del soporte.

SÉPTIMO: ampliar los espacios donde se aplica el Método. Preguntarse siempre: ¿dónde y cuándo es importante actuar de modo Paideia? En reuniones, seguramente, pero no solamente. Trabajar juntos, estimulando espacios de reflexión. Ofertar experimentando. Unir la modalidad del soporte con la del grupo. Antes, durante y después del hacer, analizar, reflexionar en base a los resultados, en base a la práctica. Ese hacer reflexivo es muchas veces un modo eficaz para quebrar resistencias e inseguridades que el diálogo teórico jamás resolvería.

En este caso, alcanzar el análisis de relaciones de poder y de afecto depende tanto de la capacidad de escucha de otros discursos como de observar a otros realizando o sufriendo impactos de la práctica. Para ampliar la capacidad de análisis de los grupos es fundamental que aprendan a escuchar, a observar el resultado de sus intervenciones y a leer textos teóricos, metodológicos o de relatos de experiencias.
OCTAVO: Autorizar a los grupos a ejercer la crítica generosa y a desear cambios: las instituciones controlan personas, desautorizándolas a tomar iniciativas que no estén previstas en los reglamentos. El funcionamiento psíquico y las ideologías bloquean el deseo y hasta la comprensión de sí mismos y de las relaciones sociales. La Oferta de temas, o de fragmentos de análisis, buscan ampliar la capacidad de las agrupaciones de lidiar con estos impedimentos: la tarea de colocar en análisis temas sagrados, no-dichos, relaciones encubiertas, derechos no ejercidos, etc.

NOVENO: Autorizarse a ser agente directo y no solamente soporte de equipos: el dirigente, el profesional y aún un miembro del grupo forman parte del Colectivo al cual quieren dar soporte. Aquel que realiza un soporte institucional específico se vincula al colectivo durante un cierto período y después se va. En este sentido, tiene como función central valerse del método para trabajar con el grupo. Los otros no pueden actuar todo el tiempo en base a la metodología Paideia, hay momentos en que el dirigente ejerce acciones administrativas, el profesional realiza sus tareas respectivas. En este sentido, existen espacios de acción en los cuales cumplirán sus funciones específicas sin la necesidad de ruedas previas o de espacios en donde estén discutiendo todo a cada momento, aun cuando la evaluación y el desdoblamiento de estos actos puedan constituirse en temas de las ruedas.

Un ejemplo: un dirigente pretende controlar una epidemia de dengue. ¿Cómo efectuar el combate al problema según la perspectiva del Soporte Paideia?

Primero, valerse del Método Paideia, recordando que existen otras posibilidades para hacer cosas. Y ¿qué es el Método? Pasa por la capacidad de analizar, reconocer la red de poder, de afectos, el compromiso con valores (finalidades) y con campos de conocimiento. En este sentido, el dirigente debe reconocerse como alguien distinto al equipo de técnicos o usuarios. No puede renunciar a su lugar de poder, negar la jerarquía, la diferencia de roles; supuestamente, él tiene un compromiso con los resultados, un tiempo definido por factores universales, 
externos en relación al grupo de técnicos, diferente de otros agentes implicados. Para el Método no existe neutralidad: el mejor remedio para atenuar los conflictos con este tipo de factores es explicitándolos, dejar claro desde dónde se está hablando, con qué objetivo, qué se busca, con qué velocidad, etc.

Mientras tanto, se debe actuar según el concepto de triple objetivo en cualquier emprendimiento social: buscar valores de uso -controlar la epidemia-, pero asegurar el Efecto Paideia sobre los sujetos comprometidos con el proyecto y con la práctica. Para esto, es fundamental construir el proyecto -diagnóstico de situación y definición de tareas- según la metodología Paideia. O sea, tomar también como finalidad que el equipo y los usuarios se apropien de un modo de pensar y de accionar que procure conocer $y$ modificar tanto el ambiente como a los propios sujetos comprometidos.

¿Y el tiempo de control de la epidemia? ¿Será equivalente al de la constitución de nuevos sujetos? No, con certeza: nunca corresponden claramente. Lo que significa, en alguna medida, que siempre se podrá crear algún tipo de compromiso o de contrato entre las distintas perspectivas. Para adecuar estos tiempos, le cabe al dirigente valerse de su poder constituido para acercar Ofertas: presentar las modalidades de acción contra el dengue considerados tradicionales, aportar informaciones sobre las presiones políticas, análisis sobre el riesgo de sufrimiento y muerte, etc. Pero también abrir espacios, colocando en análisis afectos y relaciones de poder.

La reunión es un espacio de análisis, lo otro es el hacer. Estimular al equipo para que arme ruedas con usuarios, para que agrupaciones armen ruedas con otras agrupaciones, experimentando, ellos también, el Método. Y actuar según las posibilidades, no esperar el gran día porque nunca llegará, ijamás! Y si por casualidad Ilega, con seguridad, sólo nos daremos cuenta muchos años después, en general, lamentando lo que no supimos reconocer $y$ aprovechar.

Por último: todo Soporte mínimamente sabio descubre que sólo se consigue ser soporte cuando nos autorizamos a que el grupo a quien pretendemos ayudar sea nuestro soporte. Un buen dirigente dirige y es dirigido, comanda y es comandado por aquellos con los que trabaja.

\section{REFERENCIAS BIBLIOGRÁFICAS}

1. Coriat B. El taller y el cronómetro: ensayo sobre el taylorismo, el fordismo y la producción en masa. Madrid: Siglo Veintiuno; 1992.

2. Morin E. Meus Demônios. Rio de Janeiro: Bertrand Brasil; 2003.

3. Campos GW. Um método para análise e cogestão de coletivos. São Paulo: Hucitec; 2000.
4. Jaeger W. Paidéia: a formação do homem grego. São Paulo: Martins Fontes/UnB; 1986.

5. Gramsci A. Concepção dialética da história. Rio de Janeiro: Civilização Brasileira; 1978.

6. Onocko Campos R. O planejamento no Labirinto: uma viagem hermenêutica. São Paulo: Hucitec; 2003. 